\title{
Beam splitting in chiral nematic liquid crystals
}

\author{
Filip A. Sala* \\ Faculty of Physics, Warsaw University of Technology, Koszykowa 75, 00-662 Warsaw, Poland
}

Received November 28, 2018; accepted December 31, 2018; published December 31, 2018

\begin{abstract}
By lunching a beam into chiral nematic liquid crystals it is possible to achieve a non-diffractive beam similar to a soliton. This effect is caused by molecular reorientation i.e. nonlinear response of the material forming the areas of higher refractive index. Diffraction is suppressed by the focusing effect. For appropriate launching conditions it is also possible to achieve a beam which splits into two or more separate beams. Such a phenomenon is discussed in this article and analyzed theoretically. To model this effect the Fully Vectorial Beam Propagation Method coupled with the Frank-Oseen elastic theory is used. Simulations are performed for various input beam powers, widths, polarization angles and launching positions.
\end{abstract}

Nematic liquid crystals are anisotropic materials which possess the ability to react to external electric fields. In the presence of an electric field, molecular reorientation occurs which leads to a change of refractive indexes along ordinary and extraordinary directions. The light beam can be a source of such an electric field. In nematic liquid crystals the propagation of non-diffractive beams, called nematicons, was already observed [1-2].

Many works have been done to date including logic gates [3], diodes [4], light steering [5-6], cell capacitance [7], discrete diffraction etc. The influence of thermal effects on molecular reorientation was also analyzed [8-9]. In Chiral Nematic Liquid Crystals (ChNLCs) an appropriate dopant is added to achieve a natural twist along the axis. The concentration of a dopant influences the length at which molecules rotate by 360 degrees and is called a pitch [10-11]. In such a structure, a non-diffractive beam, like a soliton, can be observed [12]. Switching and bistability of such beams was investigated [13]

In this article, the phenomenon of beam splitting in chiral nematic liquid crystals is presented. Such a phenomenon was already analyzed and observed experimentally for tilt reorientation and an externally applied field [14]. On the other hand, in the following article the phenomenon is analyzed theoretically, and beam propagation is modeled with vectorial methods combined with an elastic theory taking into account two angles to describe molecular orientation. The theoretically analyzed setup (Fig. 1) consists of a cell filled with chiral

*E-mail: filip.sala@pw.edu.pl nematic liquid crystals. The cell is $H=32 \mu \mathrm{m}$ high and $W=80 \mu \mathrm{m}$ wide. The pitch of a cholesteric is $16 \mu \mathrm{m}$, so there are two full twists around the chiral axis inside the cell. The phenomenon can occur for different values of pitch and even a different number of full twists inside the cell. In such a case, quantitative results can be different but qualitative results will be the same.

The molecular orientation is described by two angles $\varphi$ and $\theta$ (see Fig. 1). At all the boundaries there are strong anchoring conditions $\varphi(x=0)=\pi / 2$ and $\varphi(x=H)=9 / 2 \pi$ and $\theta(x=0)=\pi / 2$ and $\theta(x=H)=\pi / 2$. The $y$-polarized beam of a Gaussian shape is launched into the cell. Parameter $\Delta x$ describes the shift of the light beam launching position with respect to the center of the cell.

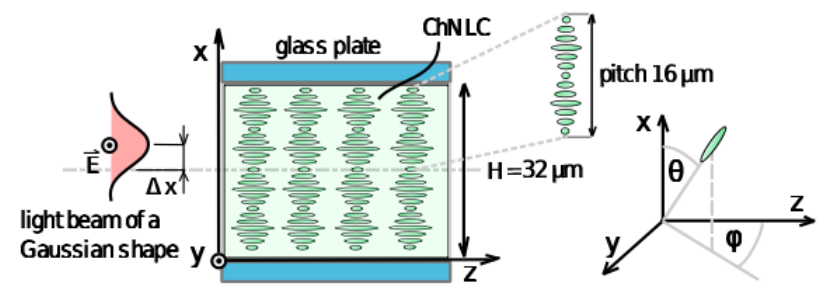

Fig. 1. The analyzed setup and the coordinate system.

Beam propagation was modeled using the Fully-Vectorial Beam Propagation Method (FV-BPM) [15] derived directly from Maxwell's equations. A theory based on Frank-Oseen's equation was used to simulate molecular reorientation i.e. the nonlinear response of a liquid crystal. More information and details about the used methods can be found in [16-17].

The resolutions of the performed simulations are $\mathrm{d} x=$ $\mathrm{d} y=0.25 \mu \mathrm{m}$ and $\mathrm{d} z=0.01 \mu \mathrm{m}$ and the wavelength is equal to $\lambda=532 \mathrm{~nm}$. The material parameters correspond to the parameters of a liquid crystal called 1110. The electric permittivities are $\varepsilon_{\|}=2.262, \varepsilon_{\perp}=2.134$ and Frank elastic constants are $\mathrm{K}_{11}=\mathrm{K}_{33}=21.39 \mathrm{pN}$ and $\mathrm{K}_{22}=8 \mathrm{pN}$. Simulations were performed for various beam powers, widths, polarizations and launching positions of the beam. If not otherwise stated, the simulations were performed on a distance of $1500 \mu \mathrm{m}$ for the beam of an initial width $\mathrm{FWHM}=4 \mu \mathrm{m}$ (Full Width at Half Maximum). 


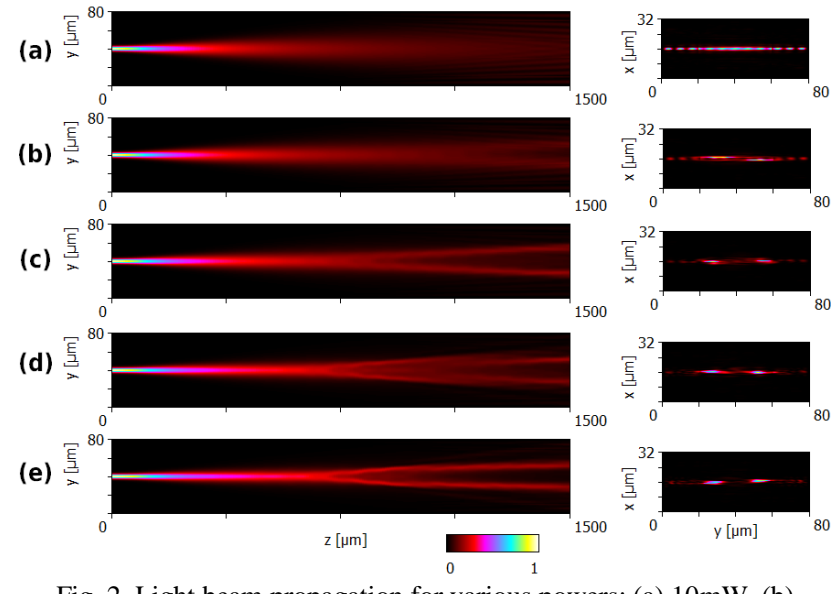

Fig. 2. Light beam propagation for various powers: (a) $10 \mathrm{~mW}$, (b) $30 \mathrm{~mW}$, (c) $50 \mathrm{~mW}$, (d) $75 \mathrm{~mW}$, (e) $100 \mathrm{~mW}$. The width of the beam is $\mathrm{FWHM}=4 \mu \mathrm{m}$. Light intensity distributions in $y z$ plane (on the left) and a cross section at $\mathrm{z}=1500 \mu \mathrm{m}$ (on the right).

In Fig. 2 the distribution of light intensity versus input beam power is presented. For low powers the beam strongly diffracts. However, for powers lower than those needed to form a non-diffractive beam some splitting of light energy occurs, in such a case the propagation is dominated by diffraction and separate beams cannot be observed. By increasing the power, a non-diffractive beam (similar to a soliton) can be formed. It appears that for powers which are sufficient to excite a non-diffractive beam, light splitting can occur, like it is presented in Fig. $2 \mathrm{c} \div \mathrm{e}$. This phenomenon is caused by the symmetry of electric field distribution of a light beam with respect to the surrounding chiral structure. This stationary solution is meta-stable and even a slight change of launching conditions can suppress beam splitting.

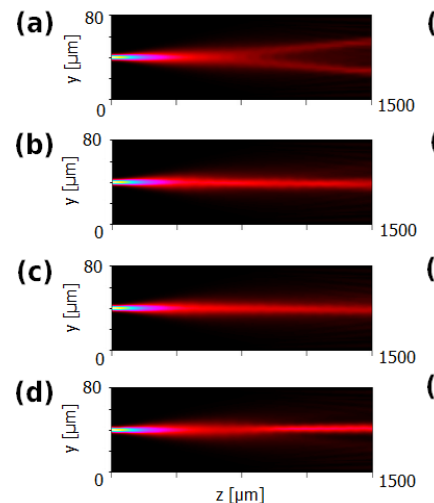

$\mathrm{z}[\mu \mathrm{m}]$

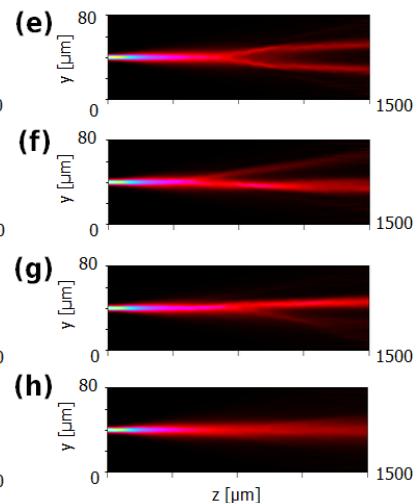

$\mathrm{z}[\mu \mathrm{m}]$
Fig. 3. Light beam propagation for an input beam power of $50 \mathrm{~mW}$ $(\mathrm{a} \div \mathrm{d})$, and $100 \mathrm{~mW}(\mathrm{e} \div \mathrm{h})$ for various shifts of the launching position: (a, e) $\Delta \mathrm{x}=0 \mu \mathrm{m},(\mathrm{b}, \mathrm{f}) 0.25 \mu \mathrm{m},(\mathrm{c}, \mathrm{g}) 0.5 \mu \mathrm{m}(\mathrm{d}, \mathrm{h}) 1 \mu \mathrm{m}$. Simulations performed for a beam of $\mathrm{FWHM}=4 \mu \mathrm{m}$.

Simulations taking into account the changes in the launching position along the $x$-axis, are presented in
Fig. 3. For a $50 \mathrm{~mW}$ beam, the change in the launching position, even as small as $\Delta x=0.25 \mu \mathrm{m}$, leads to the formation of a single, straight non-diffractive beam. For higher powers, i.e. $100 \mathrm{~mW}$ and the shift of $\Delta x=0.25 \mu \mathrm{m}$ or even $\Delta x=0.5 \mu \mathrm{m}$, the beam still splits into two beams, however the energy of the light travelling in each branch is uneven. Like in the previous case, for higher values of $\Delta x$ a single non-diffractive beam is formed. Even small changes of the launching position significantly influence beam propagation because the electric field corresponding to the launched beam is no longer symmetric with respect to the center of the cell and a stable solution can be found. Independent simulations were also performed to determine the influence of the shift of the launching position along the $y$ axis. It appears that such a shift does not influence light propagation as the structure is homogeneous along the $y$ axis. Even strong anchoring conditions at $y=0$ and $y=W$, do not change the propagation, as long as the beam is launched far from the boundary.

The influence of the beam width was also analyzed. It appears that changes of the width do not suppress the splitting of the beam, as far as the diffraction can be balanced by the focusing caused by nonlinear effects. In other words, as far as it is possible to form a nondiffractive beam, it is also possible to achieve a splitting beam. Moreover, it is also possible to obtain splitting into more than two beams, by decreasing the input beam width, like it is shown in Fig. 4. In such a case, diffraction is stronger and the beam spreads along the y-axis forming more distinct breathing beams.

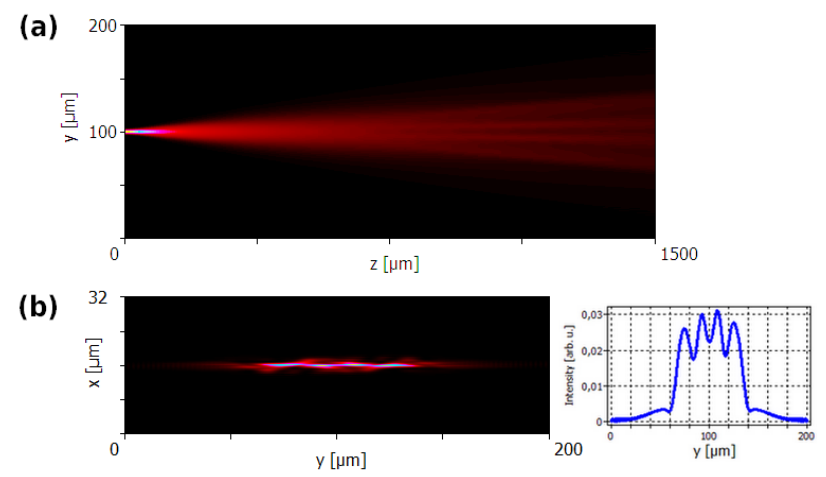

Fig. 4. Light propagation in a larger cell of $32 \mu \mathrm{m} \times 200 \mu \mathrm{m}$. Simulation performed for $\mathrm{FWHM}=2.5 \mu \mathrm{m}$ and input beam power $\mathrm{P}=100 \mathrm{~mW}$. (a) $y z$ plane (b) cross section at the end of the cell.

In all the previous simulations the input beam was $y$ polarized. Some simulations for polarization angles of $22.5^{\circ}$ and $45^{\circ}$, measured from the $y$-axis, were also carried out. However, for $22.5^{\circ}$ it was still possible to achieve beam splitting, for a polarization of $45^{\circ}$ such a phenomenon was not observed. It was only possible to 
achieve non-diffractive beams for powers higher than those for $y$-polarized beams. The higher the polarization angle, the more difficult it is to observe beam splitting. It is caused by the fact that it is much more difficult to obtain a non-diffractive beam as more power is needed, and due to a saturative nature of liquid crystals nonlinearity.

Concluding, it is possible to achieve beam splitting in chiral nematic liquid crystals. The width of the input beam does not influence the phenomenon as long as it is possible to achieve a non-diffractive beam. For the best results the input beam should be y-polarized. Increasing the polarization angle increases the minimum power needed to obtain a non-diffractive beam and thus it is much more difficult to achieve beam splitting. The phenomenon is very sensitive to the launching position along the $x$ axis (chiral axis). Even slight changes can suppress the beam splitting effect. On the other hand, changes along the $y$ axis do not influence the propagation as long as the beam is far from the boundary.

All images presenting simulations were encoded with grayscale print-friendly false color palettes [18].

This work was supported by the grant of the Faculty of Physics, Warsaw University of Technology.

\section{References}

[1] G. Assanto, M.A. Karpierz, Liq. Cryst. 36, 1161 (2009).

[2] G. Assanto, Nematicons: Spatial Optical Solitons in Nematic Liquid Crystals (John Wiley \& Sons Inc. Hoboken, New Jersey 2013).

[3] A. Piccardi, A. Alberucci, U. Bortolozzo, S. Residori, G. Assanto, Appl. Phys. Lett. 96, 071104 (2010).

[4] D. Melo, I. Fernandes, F. Moraes, S. Fumeron, E. Pereira, Phys. Lett. A 380, 3121 (2016)

[5] U. Laudyn, M. Kwaśny, F.A. Sala, M.A. Karpierz, N.F. Smyth, G. Assanto, Sci. Rep. 7, 12385 (2017).

[6] M. Kwaśny, U.A. Laudyn, F.A. Sala, A. Alberucci, M.A. Karpierz, G. Assanto, Phys. Rev. A 86, 013824 (2012).

[7] F.A. Sala, M.M. Sala-Tefelska, J. Opt. Soc. Am. B. 35, 133 (2018).

[8] U.A. Laudyn, A. Piccardi, M. Kwasny, M.A. Karpierz, G. Assanto, Opt. Lett. 43, 2296 (2018).

[9] F.A. Sala, M.M. Sala-Tefelska, M.J. Bujok, J. Nonlinear Opt. Phys. Mater. 27, 1850011 (2018).

[10] I.-C. Khoo, Liquid crystals (John Wiley \& Sons, Inc 2007).

[11] P.G. de Gennes, J. Prost, The Physics of Liquid Crystals (Clarendon Press 1995)

[12] U.A. Laudyn, P.S. Jung, M.A. Karpierz, G. Assanto, Sci. Rep. 6, 22923 (2016).

[13] J. Beeckman, A. Madani, P.J.M. Vanbrabant, P. Henneaux, S-P. Gorza, M. Haelterman, Phys. Rev. A 83, 033832 (2011)

[14] A. Madani, J. Beeckman, K. Neyts, Opt. Comm. 298-299, 222 (2013).

[15] G.D. Ziogos, E.E. Kriezis, Opt. Quant. Electron. 40, 10 (2008).

[16] F.A. Sala, M.A. Karpierz, J. Opt. Soc. Am. B 29, 1465 (2012).

[17] F.A. Sala, M.A. Karpierz, Opt. Expr. 20, 13923 (2012).

[18] F.A. Sala, Displays 46, 9 (2017). 